\title{
CHARACTERIZATION OF AMINOGLYCOSIDE-LIPID INTERACTIONS AND DEVELOPMENT OF A REFINED MODEL FOR OTOTOXICITY TESTING
}

\author{
Barbara M. Wang, ${ }^{*}$ Norman D. Weiner,${ }^{*} \dagger$ Akira TAKada $\ddagger$ and Jochen SchachT $\ddagger$ \\ ${ }^{*}$ College of Pharmacy and $\ddagger$ Kresge Hearing Research Institute, University of Michigan, Ann Arbor, \\ MI 48109 , U.S. $\Lambda$.
}

(Received 26 November 1983; accepted 21 February 1984)

\begin{abstract}
Aminoglycoside interactions with various phospholipids were measured in three model systems and compared with the ototoxicities of the drugs: (a) competition for $\left[{ }^{14} \mathrm{C}\right]$ neomycin binding; (b) competition for ${ }^{45} \mathrm{Ca}^{2+}$ binding; and (c) cffect on surface pressurc of monomolecular lipid films. The efficacies of the antibiotics in displacing neomycin from phosphatidylserine, phosphatidylinositol or phosphatidylinositol bisphosphate were netilmicin $>$ neomycin $\geq$ gentamicin; the efficacies in displacing calcium from phosphatidylinositol, phosphatidylinositol phosphate or phosphatidylinositol bisphosphate were netilmicin $>$ gentamicin $>$ neomycin $\gg$ kanamycin $>$ spectinomycin. Neither measure correlated well with the ototoxicities of the drugs which were quantitated at equimolar drug concentrations in cochlear perfusions: neomycin $>$ gentamicin $\geq$ tobramycin $>$ netilmicin $\geq$ amikacin. When monomolecular films of phosphatidylcholine with phosphatidylserine, cardiolipin, phosphatidylinositol, or phosphatidylinositol phosphate or bisphosphate were challenged with neomycin, the phosphatidylinositol bisphosphate film showed a unique dose-dependent increase in surface pressure while the others showed a decreasc or no significant $\mathrm{cffect}$. The abilities of aminoglycosides to increase the surface pressure of a film of phosphatidycholine : phosphatidylinositol bisphosphate $(1: 1 \mathrm{molar}$ ratio) in the presence of $3 \mathrm{mM} \mathrm{CaCl}_{2}$ correlated well with their toxicities. Non-ototoxic cations increased the film pressure or left it unaffected. The results confirm the unique interactions between aminoglycosides and phosphatidylinositol bisphosphate as a possible basis of a mechanism of toxicity and development of a drugscreening system.
\end{abstract}

The ototoxicity of aminoglycosides is of special clinical significance because of the potential for permanent loss of inner ear function. Symptomatic auditory and vestibular ototoxicity occurs in about $2 \%$ [1] and asymptomatic ototoxicity occurs in about $10 \%[2,3]$ of treated patients. Almost 40 years after the discovery of this class of powerful antibacterial agents, their ototoxicity is still not understood at the molecular level and neither is their nephrotoxicity.

A wide variety of aminoglycoside-induced alterations of cell metabolism has been reported, leading to hypotheses or model systems that attempt to explain the mechanisms of oto- or nephrotoxicity. These model systems generally rely on a primary interaction between an aminoglycoside and a "target" compound such as muccopolysaccharides [4], phospholipids $[5,6]$ or even anionic dyes [7].

A number of independent studies on cochlear and renal lipid metabolism and on physicochemical models now strongly implicate anionic phospholipids as receptors for aminoglycosides $[8,9]$. Furthermore, our previous work on phospholipid-aminoglycoside interactions in vivo [10] and in vitro [11] suggests that the polyphosphoinositides are specific binding sites, and a monomolecular film of these lipids was introduced as a model for ototoxicity testing $[5,12]$. A number of points, however, remained unresolved:

(1) What is the relative importance of phosphatidylinositol phosphate and bisphosphate with

t Author to whom all correspondence should be addressed. respect to the interaction of "polyphosphoinositides" with aminoglycosides?

(2) Will measurements of drug binding or calcium displacement with isolated lipids show toxicityrelated interactions in vitro or are more complex experimental conditions required, e.g. those which measure resultant physicochemical membrane changes?

(3) Can a model system be devised to differentiate toxicities accurately and independently of the magnitude of the positive charge of the drugs?

We have attempted to refine our model in order to clarify the above issues and to test critically the postulate of phosphatidylinositol bisphosphate as a receptor site for aminoglycosides.

\section{MATERIALS AND METHODS}

Phosphatidylserine, phosphatidylinositol, and cardiolipin were purchased from the Sigma Chemical Co., St. Louis, MO. Phosphatidylcholine was purified from crude egg lecithin on an alumina column. Phosphatidylinositol phosphate and phosphatidylinositol bisphosphate were prepared from Sigma brain extract by chromatography on immobilized neomycin [13]. Neomycin sulfate, reagent grade, was purchased from the Sigma Chemical Co.; gentamicin and netilmicin sulfate were gifts from the Schering Corp., Bloomfield, NJ; tobramycin was from the Eli Lilly Co., Indianapolis, IN, and amikacin from Bristol Laboratories, New York, NY; neamine and methylneobiosamine were from the 
Upjohn Co., Kalamazoo, MI. $\left[\mathrm{G}-{ }^{14} \mathrm{C}\right]$ Neomycin was a gift of Dr. G. Flynn, College of Pharmacy, University of Michigan.

The water used was thrice distilled, the second and third time in an all-glass apparatus. The buffer in all the experiments was 0.05 M HEPES ( N-2-hydroxyethyl-piperazine- $N^{\prime}$-2-sulfonic acid), $\mathrm{pH} 7.0$, ionic strength 0.2 . The sodium chloride used to adjust the ionic strength was heated previously for $12 \mathrm{hr}$ at $700^{\circ}$. Phosphatidylinositol phosphate and phosphatidylinositol bisphosphate were stored dried; other lipids were kept in chloroform: methanol $(95: 5, v / v)$ in the dark under nitrogen at $-20^{\circ}$. The lipids were examined prior to use by TLC and estimated to be at least $95 \%$ pure.

Binding studies were carried out in a biphasic (chloroform: water) system. The aqueous phase was $200 \mu \mathrm{l}$ of $187 \mathrm{mM} \mathrm{NaCl}$ in $50 \mathrm{mM}$ sodium HEPES, $\mathrm{pH} \mathrm{7.4}$; the organic phase was $200 \mu \mathrm{l}$ of $0.1 \mathrm{mM}$ lipid in chloroform:methanol $(9: 1)$. For neomycin displacement, $\left[{ }^{14} \mathrm{C}\right]$ neomycin $(1.3 \mathrm{mCl} / \mathrm{mmole})$ and the competing aminoglycoside were added at concentrations of $0.1 \mu \mathrm{M}$ to $1 \mathrm{mM}$. For calcium displacement, ${ }^{45} \mathrm{Ca}^{2+}$ was included in the aqueous phase at $0.1,0.125,0.17,0.25,0.5$ and $1 \mathrm{mM}$ and the competing aminoglycosides at $0.1 \mu \mathrm{M}$ to $1 \mathrm{mM}$. Radioactive neomycin or calcium in the chloroform phase was considered as lipid-bound, and blanks without lipid served as controls. Analyses were by Lineweaver-Burk and Scatchard plots.

Cochlear perfusions. Perilymphatic perfusions were carried out in male albino guinea pigs (200$250 \mathrm{~g}$ ) as previously described [14]. In brief, glass capillaries were implanted into the basal turns of the scala tympani and the scale vestibuli of the cochlea after a conventional ventral surgical approach. Artificial perilymph with or without $10 \mathrm{mM}$ aminoglycoside was perfused for $60 \mathrm{~min}$ at a rate of approximately $30 \mu \mathrm{l} / \mathrm{min}$. The cochlear microphonic potential was monitored continuously via a recording electrode in the scala tympani in response to broadband noise $(50 \mathrm{~Hz}$ to $10 \mathrm{kHz})$ delivered by a calibrated earphone attached to a speculum placed directly before the tympanic membrane. An intensity of $70 \mathrm{~dB}$ normally yielded a response of $250-330 \mu \mathrm{V}$. Animals with a microphonic potential of less than $200 \mu \mathrm{V}$ were excluded from the studies.

Monolayer studies. The subphase consisted of $0.05 \mathrm{M}$ HEPES $(\mathrm{pH}=7, \mu=0.2)$, and its surface was swept clean by vacuum suction. The film-forming lipid was spread in hexane : ethanol $(95: 5, \mathrm{v} / \mathrm{v})$ to the required initial surface pressure. A stationary $5 \mathrm{ml}$ syringe, whose needle remained below the film throughout the experiment, delivered the drug into the subphase which was stirred by a Teflon-coated magnetic bar at low speed without disturbing the lipid film. The surface pressure was measured by the Wilhelmy plate method, with a roughened platinum plate suspended from a torsion balance which was sensitive to surface pressure changes of $0.1 \mathrm{dyn} / \mathrm{cm}$. The film was allowed to stabilize for $30 \mathrm{~min}$ before various concentrations of cationic compounds were injected into the subphase. Ten minutes of stirring preceded each surface pressure reading. Each run was in duplicate, and reproducibility was usually within 0.1 to $0.2 \mathrm{dyn} / \mathrm{cm}$.

\section{RESULTS}

Binding studies. The affinities of various aminoglycosides to phospholipids were determined by their abilities to displace $\left[{ }^{14} \mathrm{C}\right]$ neomycin. Neomycin binding to phosphatidylserine or phosphatidylinositol showed conventional Lineweaver-Burk and Scatchard plots: phosphatidylinositol, $K_{m}=$ $229 \pm 68 \mu \mathrm{M}$; phosphatidylserine, two sites $K_{m_{l}}=$ $59 \pm 1 \mu \mathrm{M}, K_{m l}=265 \pm 120 \mu \mathrm{M}$. Neomycin binding to phosphatidylinositol bisphosphate was complex, indicating positive conperativity similar to ohservations made for calcium binding (below). Relative binding affinities were therefore assessed by neomycin displacement at fixed drug concentrations (Table 1). As expected, at equimolar concentration neomycin (USP grade) displaced about $50 \%$ of the radiolabeled compound. With phosphatidylserine and phosphatidylinositol, netilmicin was significantly more effective than gentamicin or neomycin. With phosphatidylinositol bisphosphate the three drugs were equally effective.

Calcium displacement. Calcium binding to phosphatidylinositol and phosphatidylinositol phosphate showed a single binding site, each with $K_{m}=$ $0.87 \pm 0.42 \mathrm{mM}(\mathrm{N}=17)$ and $K_{m}=0.40 \pm 0.30 \mathrm{mM}$ $(\mathrm{N}=3)$ respectively. Inhibitor constants (Table 2 ) ranked netilmicin, gentamicin, and neomycin highest while kanamycin and spectinomycin were considerably less inhibitory. Calcium binding to phosphatidylinositol bisphosphate showed positive coop-

Table 1. Displacement of $\left[{ }^{14} \mathrm{C}\right]$ neomycin from phospholipids*

\begin{tabular}{lllc}
\hline \multirow{2}{*}{$\begin{array}{l}\text { Competing } \\
\text { drug added }\end{array}$} & \multicolumn{3}{c}{$\left[{ }^{14} \mathrm{C}\right]$ neomycin (nmoles bound/umole lipid) } \\
\cline { 2 - 4 } & \multicolumn{1}{c}{$\mathrm{PhS}$} & \multicolumn{1}{c}{ PhI } & $\mathrm{PhIP}_{2}$ \\
\hline None & $94 \pm 13(100 \%)$ & $50 \pm 6(100 \%)$ & $5.2 \pm 2.4(100 \%)$ \\
Neomycin & $56 \pm 9(59 \%)$ & $26 \pm 3(52 \%)$ & $2.4 \pm 1.3(46 \%)$ \\
Gentamicin & $60 \pm 8(64 \%)$ & $22 \pm 3(44 \%)$ & $2.5 \pm 0.5(48 \%)$ \\
Netilmicin & $43 \pm 8 \dagger(46 \%)$ & $15 \pm 3+(30 \%)$ & $2.4 \pm 1.1(46 \%)$ \\
\hline
\end{tabular}

* Binding of $\left[{ }^{14} \mathrm{Clneomycin}(0.5 \mathrm{mM})\right.$ was determined as described in Materials and Methods. All drugs were added at $0.5 \mathrm{mM}$. Numbers are means \pm S.D. of five determinations each. Abbreviations: PhS, phosphatidylserine; PhI, phosphatidylinositol; and PhIP $_{2}$, phosphatidylinositol bisphosphate.

+ Differs from gentamicin $(P<0.01)$ and neomycin $(P=0.04)$.

$\ddagger$ Differs from gentamicin and neomycin $(\mathrm{P}<0.01)$. 
Table 2. Effects of aminoglycosides on calcium binding to phospholipids*

\begin{tabular}{lccrr}
\hline & \multicolumn{2}{c}{$K_{i}(\mu \mathrm{M})$} & & $K_{D 50}(\mu \mathrm{M})$ \\
\cline { 2 - 3 } \cline { 5 - 5 } Competing drug & PhI & PhIP & & $\mathrm{PhIP}_{2}$ \\
\hline Netilmicin & $6 \pm 3$ & $9 \pm 11$ & & 10 \\
Gentamicin & $30 \pm 20$ & $7 \pm 2$ & & 31 \\
Neomycin & $40 \pm 25$ & $53 \pm 37$ & & 44 \\
Kanamycin & $363 \pm 208$ & $328 \pm 276$ & & 3000 \\
Spectinomycin & $1772 \pm 11$ & $411 \pm 24$ & & $>3000$ \\
\hline
\end{tabular}

* Calcium binding was determined as described in Materials and Methods. $K_{i}$ values are the means \pm S.D. of three to five determinations each. $K_{D 50}\left(\right.$ at $1 \mathrm{mM} \mathrm{Ca}^{2+}$ ) is extrapolated by linear regression analysis from two experiments at three different drug concentrations each. Abbreviations: PhI, phosphatidylinositol; PhIP, phosphatidylinositol phosphate; and $\mathrm{PhIP}_{2}$, phosphatidylinositol bisphosphate.

erativity and multiple binding sites (details to be published elsewhere). Displacement of calcium was determined as $K_{D 50}$, i.e. the concentration of a drug necessary to displace $50 \%$ of calcium at $1 \mathrm{mM} \mathrm{Ca}^{2+}$. The rank order of inhibition was essentially the same as with the other lipids.

Monomolecular films. Interaction between neomycin and various purified acidic phospholipids in monomolecular films were investigated previously by Lodhi et al. [12]. We now tested mixtures of various acidic phospholipids with phosphatidylcholine to examine how the acidic phospholipids behave upon addition of calcium and neomycin in the presence of a neutral phospholipid.

The choice of experimental parameters $(1: 1$ mole ratio of phosphatidylcholine:acidic phospholipid,

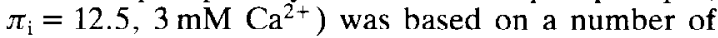
pilot experiments. While most of the pure phospholipids produced stable monolayers, phosphatidylinositol bisphosphate monolayers were unstable (desorbed into the subphase) and required at least an equimolar amount of egg lecithin to maintain physical stability. Phosphatidylcholine was chosen as the neutral phospholipid since its film does not change pressure upon addition of neomycin (Fig. 1). Mixed films of phosphatidylcholine and phosphatidylinositol bisphosphate at a higher molar ratio of $2: 1$ gave essentially similar results, but lacked sensitivity, i.e. showed a reduced magnitude of interaction with aminoglycosides. An initial surface pressure $\left(\pi_{i}\right)$ of $12.5 \mathrm{dyn} / \mathrm{cm}$ was chosen since the effect of aminoglycosides was independent of $\pi_{i}$ in the range of $9-21 \mathrm{dyn} / \mathrm{cm}$. The stabilities of the mixed lipid films were confirmed by following the changes in surface pressure of the films over the period of time required to conduct the experiments $(3 \mathrm{hr})$. All mixed lipid films maintained a constant surface pressure after equilibration.

While the effect of neomycin on the lipids was not affected drastically by the above parameters, its magnitude was dependent on $\mathrm{Ca}^{2+}$ concentration (Table 3 ). The greatest change in surface pressure was induced at $3 \mathrm{mM} \mathrm{Ca}{ }^{2+}$. The slight decrease in the change of surface pressure at higher $\mathrm{Ca}^{2+}$ con-

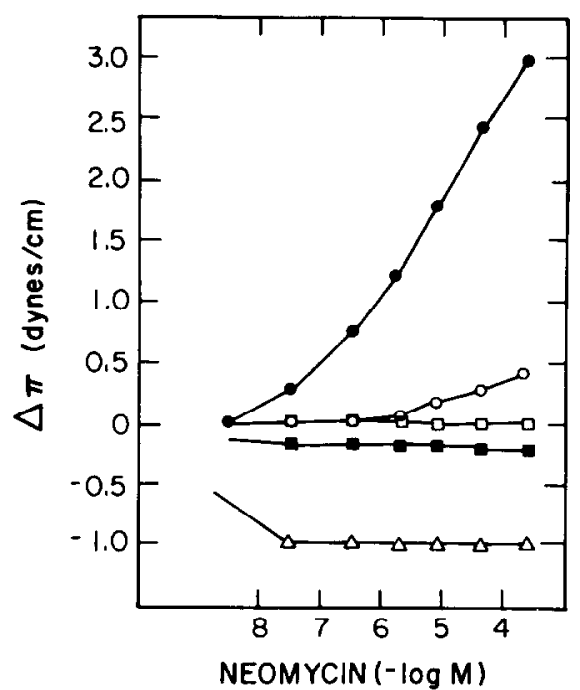

Fig. 1. Changes in surface pressure in response to neomycin. Monolayers of phosphatidylcholine: acidic phospholipid $(1: 1)$ were spread over $0.05 \mathrm{M}$ sodium HEPES, $\mathrm{pH} 7.0$, in the presence of $3 \mathrm{mM} \mathrm{CaCl}_{2}$ at $\pi_{\mathrm{i}}=$ $12.5 \mathrm{dyn} / \mathrm{cm}$. Neomycin was added, and $\pi$ was determined as described in Materials and Methods. Lipids mixed with phosphatidylcholine: ( $\sqcup-\square$ ), none or phosphatidylserine; $(\square-\square)$ cardiolipin; $(\triangle \longrightarrow \triangle)$ phosphatidylinositol; $(\bigcirc-O)$ phosphatidylinositol phosphate; and (-), phosphatidylinositol bisphosphate.

centrations is not fully understood. It is possible that the segregation of the phosphatidylinositol bisphosphate and phosphatidylcholine molecules in the film varies with the concentration of $\mathrm{Ca}^{2+}$. At $3 \mathrm{mM}$ $\mathrm{Ca}^{2+}$, the spacing between the molecules may be optimal for the interaction of neomycin with phosphatidylinositol bisphosphate.

The change in surface pressure of various anionic phospholipid-phosphatidylcholine $(1: 1)$ films was measured as a function of neomycin concentration (Fig. 1). Films of phosphatidylinositol-phosphatidylcholine, and cardiolipin-phosphatidylcholine were condensed, whereas films of phosphatidylserinephosphatidylcholine were unaffected by addition of neomycin. The surface pressure of phosphatidylinositol phosphate-phosphatidylcholine films increased slightly starting at $10^{-5} \mathrm{M}$ neomycin, whereas that of phosphatidylinositol bisphosphate-

Table 3. Effect of calcium on neomycin-induced changes of surface pressure*

\begin{tabular}{cccccc}
\hline & \multicolumn{5}{c}{ Changes of surface pressure $(\Delta \pi)$} \\
\cline { 3 - 6 } $\begin{array}{c}\text { Neomycin } \\
(\mathrm{M})\end{array}$ & $1 \mathrm{mM}$ & $2 \mathrm{mM}$ & $\begin{array}{c}\text { Calcium } \\
3 \mathrm{mM}\end{array}$ & $4 \mathrm{mM}$ & $5 \mathrm{mM}$ \\
\hline $1.6 \times 10^{-6}$ & 0.35 & 0.75 & 1.2 & 0.8 & 0.85 \\
$4.0 \times 10^{-4}$ & 0.75 & 1.8 & 2.4 & 1.8 & 1.8
\end{tabular}

* Films of phosphatidylinositol bisphosphate-phosphatidylcholine (1:1) at $\pi_{\mathrm{i}}=12.5 \mathrm{dyn} / \mathrm{cm}$ were formed in the presence of various amounts of calcium and challenged with neomycin as described in Materials and Methods. 


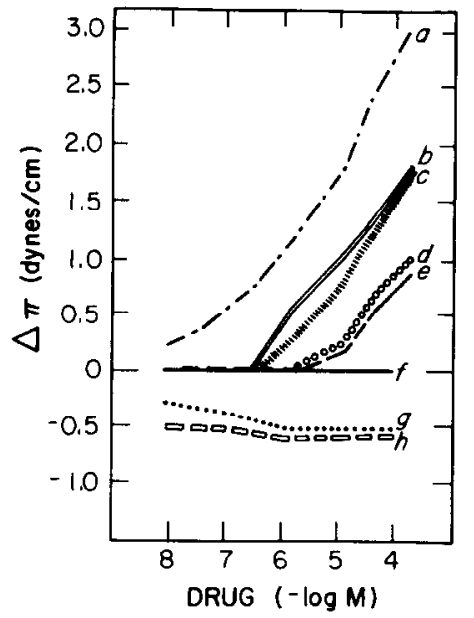

Fig. 2. Change in surface pressure induced by various aminoglycosides. Monolayers of phosphatidylcholine: phosphatidylinositol bisphosphate were spread over $0.05 \mathrm{M}$ sodium HEPES, $\mathrm{pH} 7.0$, in the presence of $3 \mathrm{mM} \mathrm{CaCl}_{2}$ at $\pi_{\mathrm{i}}=12.5 \mathrm{dyn} / \mathrm{cm}$. Drugs were added, and surface pressure was determined as described in Materials and Methods. Key: (a) neomycin; (b) gentamicin; (c) tobramycin; (d) netilmicin; (e) amikacin; (f) spectinomycin; (g) neamine and (h) methylneobiosamine. Data points are not shown for the sake of clarity. Each curve represents at least ten data points.

phosphatidylcholine films increased greatly starting at very low neomycin concentrations $\left(10^{-8} \mathrm{M}\right)$.

Next, the change in surface pressure of phosphatidylinositol bisphosphate-phosphatidylcholine $(1: 1)$ films was determined as a function of aminoglycoside concentration (Fig. 2). The two non-ototoxic fragments of neomycin, neamine and methylneobiosamine, condensed the film. All aminoglycosidic antibiotics tested, with the exception of spectinomycin, expanded it to some degree. Spectinomycin, a clinically used aminocyclitol drug for treating gonorrhea, is not considered to be ototoxic [15]. Neomycin, highly ototoxic, induced the greatest increase in surface pressure, gentamicin and tobramycin an intermediate increase, while netilmicin and amikacin were the least effective. The magnitude of the individual interactions varied with the calcium concentration as discussed earlier for neomycin; the rank order of the aminoglycosides, however, remain unchanged.

Cochlear perfusions. The toxicities of these aminoglycosides were tested in cochlear perfusions. Irreversible loss of the cochlear microphonic potential is the measure of ototoxicity quantitated either by the initial rate of loss or the loss after $30 \mathrm{~min}$ of drug application (Table 4). Based on this comparison of equimolar amounts of drug, neomycin was more toxic than gentamicin and tobramycin which. in turn, were more toxic than amikacin and netilmicin. Spectinomycin affected the microphonic potential only acutely and reversibly. The recovery induced by $\mathrm{Ca}^{2+}$ was in contrast to the irreversibility of gentamicin [14] or of even the weakest toxic drug, amikacin. Spectinomycin, therefore, did not have the aminoglycoside-like toxicity to the cochlear microphonic potential.
Table 4. Effects of aminoglycosides on cochlear microphonic potentials*

\begin{tabular}{lccr}
\hline Drug & \multicolumn{3}{c}{ Loss of microphonic potential } \\
\hline Experiment A & $\begin{array}{c}\text { Initial rate } \\
(\% / \text { min })\end{array}$ & $\begin{array}{c}\text { Loss at } 30 \mathrm{~min} \\
(\%)\end{array}$ & $\mathrm{N}$ \\
\hline None & $0.16 \pm 0.12$ & $3 \pm 3$ & 8 \\
Neomycin & $5.37 \pm 1.44$ & $54 \pm 2$ & 3 \\
Gentamicin & $3.40 \pm 1.32$ & $49 \pm 13$ & 15 \\
Tobramycin & $2.50 \pm 1.20$ & $50 \pm 13$ & 5 \\
Netilmicin & $1.69 \pm 0.55$ & $37 \pm 10$ & 5 \\
Amikacin & $1.25 \pm 0.58$ & $25 \pm 11$ & 6 \\
\hline Experiment B & CM at 30 min & CM after wash & $\mathrm{N}$ \\
& \multicolumn{4}{c}{$(\%$ loss $)$} \\
\hline Amikacin & $32 \pm 10$ & $31 \pm 9$ & 3 \\
Spectinomycin & $22 \pm 7$ & $8 \pm 11$ & 3 \\
\hline
\end{tabular}

* Perfusions were carried out as described in Materials and Methods. Experiment A: After an initial control period of $30 \mathrm{~min}$, drugs $(10 \mathrm{mM}$, in artificial perilymph) were perfused for $30 \mathrm{~min}$. Experiment B: As A, but after drug perfusion artificial perilymph with $10 \mathrm{mM} \mathrm{Ca}^{2+}$ was perfused for $30 \mathrm{~min}$ (wash) [14]. Numbers are means \pm S.D. of $\mathrm{N}$ experiments.

\section{DISCUSSION}

Several criteria have to be met by a viable model system of drug action.

First, the model should be representative of the primary membrane action rather than of a secondary effect, such as delayed interactions with nucleic acid [16] or glycolytic enzymes [17].

Second, the model should incorporate the chemical uniqueness of the affected organs, i.e. in case of aminoglycoside toxicity, the inner ear and kidney Studies based on the interaction of aminoglycosides with ubiquitous cellular components such as nucleic acids, glycolytic enzymes, or major phospholipids [9] do not address the question of why aminoglycosides are not toxic to the wide variety of tissues containing these compounds. In contrast, tissue distribution of polyphosphoinositides is not uniform-high in nervous tissue and kidney [18] — and may account for the target specificity of the aminoglycosides. In the inner ear, these lipids have been demonstrated in all tissues by radioactive labeling $[10,19]$ and localized to plasma and mitochondrial membranes by immunohistochemistry [20].

Third, the model should encompass the microenvironment of the proposed "target" molecule rather than treat it as an isolated chemical entity. For instance, physiological cations such as $\mathrm{Ca}^{2+}$ should be considered since they may crucially modulate drug binding.

Fourth, the model should predict the relative ototoxicity of a series of aminoglycosides. Although a number of model studies do show some degree of correlation with clinical toxicity, it seems significant that netilmicin generally ranks as too toxic $[9,21]$, and the non-ototoxic drug fragment neamine reacts as a "toxic" aminoglycoside [6].

A biological receptor site must be considered in 
its microenvironment. This is particularly true if the binding site is part of a membrane structure and fixed within definite boundaries. Interactions of a drug with the receptor could thus lead to perturbations of the orientation, not only of the receptor proper, but of larger domains of the membrane, leading to changes in membrane fluidity and permeability. For example, a given aminoglycoside may interact with all negatively charged membrane components; the extent of perturbation of membrane structure, however, may vary and may not even directly correlate with the individual binding constants. Rather, membrane perturbations and physiological consequences will depend on how the primary interaction affects the microenvironment of the binding site. "Toxicity", therefore, may be reflected better in vitro if the model encompasses some of the complexity of the natural environment.

This concept seems clearly supported by the present study. "Simple" interactions such as drug binding or calcium displacement do not correlate with the toxicity of the drugs. Netilmicin, for example, possesses low oto- as well as nephrotoxicity, yet has a binding affinity similar to those of the highly toxic neomycin and gentamicin. This finding is essentially in agreement with a report of gentamicin binding to phospholipids and its displacement by other aminoglycosides from renal brush border membranes [9], which ranked netilmicin between the toxic neomycin and tobramycin/gentamicin. As the authors pointed out, such a rank order reflects the cationic structure of the drugs rather than their toxicities.

A similar problem of measuring charge rather than toxicity was also seen in a study of the influence of aminoglycosides on the mobility of liposomes in the absence of calcium [6]. The highly charged (+4) neamine ranked among the toxic aminoglycosides. Neamine must, however, be considered non-ototoxic since it is without effect, even when directly introduced into the inner ear [5].

The displacement of calcium represents a somewhat more complex system as it takes into consideration the presence of a physiological counterion to the acidic lipids. We had shown previously that toxic aminoglycosides and non-toxic derivatives all compete with calcium binding to synaptosomal membranes [22]. A recent study by Lüllmann and Vollmer [21], essentially confirming our earlier work on aminoglycoside-lipid interactions, also demonstrated that the diversely toxic neomycin, gentamicin, netilmicin or dibekacin all have approximately the same capability of displacing calcium from phosphatidylserine monolayers. The results presented here thus confirm and extend the notion that displacement of calcium alone is not sufficient to explain the toxicity of aminoglycosides.

The model of aminoglycoside-lipid interactions that seems to meet all criteria best is based on surface pressure changes induced in a mixed monomolecular film of lecithin and phosphatidylinositol biphosphate. The latter lipid had been identified as a primary site of drug effect in vivo $[10,19]$ and as a neomycin receptor [11]. Based on an examination of molecular models of various negatively charged phospholipids and neomycin, we had postulated that phosphatidylinositol hisphosphate should be unique in its interaction with neomycin [23]. Experimental comparison with other lipids (Fig. 1) indeed confirmed this prediction. While cations in general, as well as aminoglycosides, interact with films of anionic lipids by decreasing surface pressure, an increased pressure is seen in the case of neomycin/ phosphatidylinositol bisphosphate. This atypical interaction may be based on an electrostatic 3-point fit between the negatively charged groups of phosphatidylinositol bisphosphate and three of the positively charged amino groups of neomycin [23]. This interaction would displace calcium and orient neomycin in the film such that the resulting complex assumes an area-determining position leading to film expansion. A penetration of the drug into the film may be ruled out as it is energetically highly unfavorable because of the strong hydrophilic properties of aminoglycosides.

The rank order of aminoglycosides obtained in this system apparently yields an excellent correlation with the toxicities of the drugs quantitated by cochlear perfusion. This latter technique introduces the drugs (each at the same molar concentration) directly into the cochlea and thus measures an intrinsic (or potential) toxicity that may be higher than that determined in vivo [24]. Drug actions in vivo are influenced by renal clearance and resulting serum levels as well as by the existence of a blood-hemolabyrinthine barrier. Further complicating toxicity rankings from published in vivo observations is the fact that in most studies the aminoglycosides are administered on an equal-weight basis or in multiples of their clinical dosage. For rational structure-toxicity determinations, however, measurement of the intrinsic molecular toxicity is essential. This intrinsic molecular toxicity is obtained from cochlear perfusions (neomycin $>$ gentamicin $\geq$ tobramycin $>$ netilmicin $\geq$ amikacin) and is in good agreement with the toxicities of the drugs as extrapolated from clinical observations or in vivo animal studies [25]. Spectinomycin as well as neamine and methylneobiosamine [5] are non-ototoxic.

The refined monolayer system presented here not only accurately ranks the toxicities of aminoglycosides regardless of their charge but also clearly differentiates non-toxic cationic compounds. It is primarily the latter aspect that is most improved over the previously suggested "polyphosphoinositide" monolayer. The study also establishes that, of the two polyphosphoinositides, it is phosphatidylinositol bisphosphate which is unique in its interaction with aminoglycosides. While extrapolations from in vitro studies of monolayers to biological membranes must be made with caution, the results presented here are strongly supportive of the hypothesis that (oto)toxicity of these drugs is mediated through the presence of phosphatidylinositol bisphosphate in the membranes of affected cells. Efficient drug screening and elucidation of structure-toxicity relationships may now be based on this drug-receptor interaction.

Acknowledgements - This research was supported by Grant NS-13792 and Program Project Grant NS-05785 from the National Institutes of Health. The authors wish to acknowledge the help of Ms. Brigitte Rosinski in the binding studies. 


\section{REFERENCES}

1. G. G. Jackson, G. J. Arcieri, J. infect. Dis. 124. S130 (1971).

2. M. Barza and R. T. Scheife, Am. J. Hosp. Pharm. 34, 723 (1977).

3. C. R. Smith, K. L. Baughman, C. Q. Edwards, J. F. Rogers and P. S. Lietman, New Engl J. Med. 296, 349 (1977).

4. T. Deguchi, A. Ishii and M. Tanaka, J. Antibiot, Tokyo 31, 150 (1978).

5. S. Lodhi, N. D. Weiner, I. Mechigian and J. Schacht, Biochem. Pharmac. 29, 597 (1981).

6. P. R. Langford, E. S. Harpur, J. B. Kayes and I. Gonda, J. Antibiot., Tokyo 35, 1387 (1982).

7. H. Saito, T. Terazono and K. Nede, Ear Res. Japan 13, 108 (1982)

8. H. D. Humes, N. D. Weiner and J. Schacht, in Nephrotoxicity and Ototoxicity of Drugs (Ed. Jp. Fillastre), pp. 333-43. Editions INSERM, Paris (1982)

9. M. Sastrasinh, T. C. Knauss, J. M. Weinberg and H. D. Humes, J. Pharmac. exp. Thér, 222(2), 350 (1982).

10. A. Orsulakova, E. Stockhorst and J. Schacht, J. Neurochem. 26, 285 (1976).

11. J. Schacht, Arch Oto-Rhino-Lary. 224, 129 (1979).

12. S. Lodhi, N. D. Weiner and J. Schacht, Biochim. biophys. Acta 557, 1 (1979).
13. J. Schacht, J. Lipid Res. 19, 1063 (1978).

14. A. Takada and J. Schacht, Hear. Res. 8, 179 (1982).

15. R. R. Willcox, Br. J. clin. Pract. 29, 34 (1975).

16. J. Jarlstedt and D. Bagger-Sjöbäck, Acta oto-lar. 84 . 361 (1977).

17. M. Tachibana, O. Mizukoshi and K. Kurivama. Biochem. Pharmac. 25, 2297 (1976).

18. G. Hauser and J. J. Eichberg, Biochim. biophys. Acto 326, 201 (1973).

19. F. Stockhorst and I. Schacht, Acta oto-lar. 83, 401 (1977).

20. M. Tachibana, in Transmembrane Signalling and Sensation (Ed. F. Oosawa), Japan Scientific Societies Press, in press.

21. H. Lüllmann and B. Vollmer, Biochem. Pharmac. 31, $3769(1982)$

22. S. Lodhi, N, D. Weiner and J. Schachl, Biochim. biophys. Acta 426, 781 (1976).

23. J. Schacht, S. Lodhi and N. D. Weiner in Membrane Toxicity (Eds. M. W. Miller and A. E. Shamoo), pp 191-208. Plenum Press. New York (1977).

24. A. Anniko, A. Takada and J, Schacht, Am. J. oto-lar. 3, 422 (1982)

25. R. E. Brummett and K. E. Fox, in The Aminoglycosides (Eds. A. Whelton and H. C. Neu), pp, 419-51. Marcel Dekker, New York (1982). 